\title{
Generalized analogs of the Heisenberg uncertainty inequality
}

Ashish Bansal' and Ajay Kumar ${ }^{2 *}$

\section{${ }^{*}$ Correspondence:}

akumar@maths.du.ac.in

${ }^{2}$ Department of Mathematics,

University of Delhi, Delhi, 110007,

India

Full list of author information is

available at the end of the article

\begin{abstract}
We investigate locally compact topological groups for which a generalized analog of the Heisenberg uncertainty inequality hold. In particular, it is shown that this inequality holds for $\mathbb{R}^{n} \times K$ (where $K$ is a separable unimodular locally compact group of type I), Euclidean motion group and several general classes of nilpotent Lie groups which include thread-like nilpotent Lie groups, 2-NPC nilpotent Lie groups and several low-dimensional nilpotent Lie groups.
\end{abstract}

MSC: Primary 22E25; secondary 43A25; 22D10

Keywords: Heisenberg uncertainty inequality; nilpotent Lie group; Euclidean motion group; Plancherel formula; Fourier transform

\section{Introduction}

In 1927, Heisenberg presented a principle related to the uncertainties in the measurements of position and momentum of microscopic particles. This principle is known as Heisenberg uncertainty principle and can be stated as follows:

It is impossible to know simultaneously the exact position and momentum of a particle. That is, the more exactly the position is determined, the less known the momentum, and vice versa.

In 1933, Wiener gave the following mathematical formulation of the Heisenberg uncertainty principle:

A nonzero function and its Fourier transform cannot both be sharply localized.

Heisenberg's uncertainty inequality is a precise quantitative formulation of the above principle.

The Fourier transform of $f \in L^{1}\left(\mathbb{R}^{n}\right)$ is given by

$$
\hat{f}(\xi)=\int_{\mathbb{R}^{n}} f(x) e^{-2 \pi i(x, \xi\rangle} d x
$$

where $\langle\cdot, \cdot\rangle$ denotes the usual inner product on $\mathbb{R}^{n}$. This definition of Fourier transform holds for functions in $L^{1}\left(\mathbb{R}^{n}\right) \cap L^{2}\left(\mathbb{R}^{n}\right)$. Since $L^{1}\left(\mathbb{R}^{n}\right) \cap L^{2}\left(\mathbb{R}^{n}\right)$ is dense in $L^{2}\left(\mathbb{R}^{n}\right)$, the definition of Fourier transform can be extended to the functions in $L^{2}\left(\mathbb{R}^{n}\right)$.

The following theorem gives the Heisenberg uncertainty inequality for the Fourier transform on $\mathbb{R}^{n}$. For a proof of the theorem, see [1].

(C) 2015 Bansal and Kumar. This article is distributed under the terms of the Creative Commons Attribution 4.0 International License (http://creativecommons.org/licenses/by/4.0/), which permits unrestricted use, distribution, and reproduction in any medium, provided you give appropriate credit to the original author(s) and the source, provide a link to the Creative Commons license, and indicate if changes were made. 
Theorem 1.1 For any $f \in L^{2}\left(\mathbb{R}^{n}\right)$, we have

$$
\frac{n\|f\|_{2}^{2}}{4 \pi} \leq\left(\int_{\mathbb{R}^{n}}\|x\|^{2}|f(x)|^{2} d x\right)^{1 / 2}\left(\int_{\mathbb{R}^{n}}\|y\|^{2}|\hat{f}(y)|^{2} d y\right)^{1 / 2}
$$

where $\|\cdot\|_{2}$ denotes the $L^{2}$-norm and $\|\cdot\|$ denotes the Euclidean norm.

The Heisenberg uncertainty inequality has been established for the Fourier transform on the Heisenberg group by Thangavelu [2]. Further generalizations of the inequality on the Heisenberg group have been established by Sitaram et al. [3] and Xiao and He [4]. For some more details, see [1].

The inequality given below can be proved using Hölder's inequality and the inequality (1.1).

Theorem 1.2 For any $f \in L^{2}\left(\mathbb{R}^{n}\right)$ and $a, b \geq 1$, we have

$$
\frac{n\|f\|_{2}^{\left(\frac{1}{a}+\frac{1}{b}\right)}}{4 \pi} \leq\left(\int_{\mathbb{R}^{n}}\|x\|^{2 a}|f(x)|^{2} d x\right)^{\frac{1}{2 a}}\left(\int_{\mathbb{R}^{n}}\|y\|^{2 b}|\hat{f}(y)|^{2} d y\right)^{\frac{1}{2 b}},
$$

where $\|\cdot\|_{2}$ denotes the $L^{2}$-norm and $\|\cdot\|$ denotes the Euclidean norm.

In Section 2, we shall prove a generalized analog of the Heisenberg uncertainty inequality for $\mathbb{R}^{n} \times K$, where $K$ is a separable unimodular locally compact group of type I. In the next section, a generalized analog of the Heisenberg uncertainty inequality for the Euclidean motion group $M(n)$ is proved. The last section deals with a generalized analog of the Heisenberg uncertainty inequality for several general classes of nilpotent Lie groups for which the Hilbert-Schmidt norm of the group Fourier transform $\pi_{\xi}(f)$ of $f$ attains a particular form. These classes include thread-like nilpotent Lie groups, 2-NPC nilpotent Lie groups and several low-dimensional nilpotent Lie groups.

\section{$2 \mathbb{R}^{n} \times K, K$ a locally compact group}

Consider $G=\mathbb{R}^{n} \times K$, where $K$ is a separable unimodular locally compact group of type I. The Haar measure of $G$ is $d g=d x d k$, where $d x$ is the Lebesgue measure on $\mathbb{R}^{n}$ and $d k$ is the left Haar measure on $K$. The dual $\widehat{G}$ of $G$ is $\mathbb{R}^{n} \times \widehat{K}$, where $\widehat{K}$ is the dual space of $K$.

The Fourier transform of $f \in L^{2}(G)$ is given by

$$
\hat{f}(y, \sigma)=\int_{\mathbb{R}^{n}} \int_{K} f(x, k) e^{-2 \pi i\langle x, y\rangle} \sigma\left(k^{-1}\right) d k d x,
$$

for $(y, \sigma) \in \mathbb{R}^{n} \times \widehat{K}$.

Theorem 2.1 For any $f \in L^{2}\left(\mathbb{R}^{n} \times K\right.$ ) (where $K$ is a separable unimodular locally compact group of type I) and $a, b \geq 1$, we have

$$
\begin{aligned}
\frac{n\|f\|_{2}^{\left(\frac{1}{a}+\frac{1}{b}\right)}}{4 \pi} \leq & \left(\int_{\mathbb{R}^{n}} \int_{K}\|x\|^{2 a}|f(x, k)|^{2} d k d x\right)^{\frac{1}{2 a}} \\
& \times\left(\int_{\mathbb{R}^{n}} \int_{\widehat{K}}\|y\|^{2 b}\|\hat{f}(y, \sigma)\|_{\mathrm{HS}}^{2} d y d \sigma\right)^{\frac{1}{2 b}} .
\end{aligned}
$$


Bansal and Kumar Journal of Inequalities and Applications （2015) 2015:168

Page 3 of 15

Proof Without loss of generality, we may assume that both integrals on the right-hand side of (2.1) are finite.

Given that $f \in L^{2}\left(\mathbb{R}^{n} \times K\right)$, there exists $A \subseteq K$ of measure zero such that for $k \in K \backslash A=A^{\prime}$ (say), we have

$$
\int_{\mathbb{R}^{n}}|f(x, k)|^{2} d x<\infty
$$

For all $k \in A^{\prime}$, we define $f_{k}(x)=f(x, k)$, for every $x \in \mathbb{R}^{n}$.

Clearly, for all $k \in A^{\prime}, f_{k} \in L^{2}\left(\mathbb{R}^{n}\right)$, and for all $y \in \mathbb{R}^{n}$,

$$
\hat{f}_{k}(y)=\int_{\mathbb{R}^{n}} f(x, k) e^{-2 \pi i\langle x, y\rangle} d y=\mathscr{F} f(y, k) .
$$

By Theorem 1.1, we have

$$
\frac{n}{4 \pi} \int_{\mathbb{R}^{n}}|f(x, k)|^{2} d x \leq\left(\int_{\mathbb{R}^{n}}\|x\|^{2}\left|f_{k}(x)\right|^{2} d x\right)^{1 / 2}\left(\int_{\mathbb{R}^{n}}\|y\|^{2}\left|\hat{f}_{k}(y)\right|^{2} d y\right)^{1 / 2} .
$$

Integrating both sides with respect to $d k$, we obtain

$$
\frac{n}{4 \pi} \int_{A^{\prime}} \int_{\mathbb{R}^{n}}|f(x, k)|^{2} d x d k \leq \int_{A^{\prime}}\left(\int_{\mathbb{R}^{n}}\|x\|^{2}\left|f_{k}(x)\right|^{2} d x\right)^{1 / 2}\left(\int_{\mathbb{R}^{n}}\|y\|^{2}\left|\hat{f}_{k}(y)\right|^{2} d y\right)^{1 / 2} d k
$$

The integral on the L.H.S. is equal to $\|f\|_{2}^{2}$, so using the Cauchy-Schwarz inequality and Fubini's theorem, we have

$$
\frac{n\|f\|_{2}^{2}}{4 \pi} \leq\left(\int_{K} \int_{\mathbb{R}^{n}}\|x\|^{2}|f(x, k)|^{2} d x d k\right)^{1 / 2}\left(\int_{\mathbb{R}^{n}}\|y\|^{2} \int_{A^{\prime}}\left|\hat{f}_{k}(y)\right|^{2} d k d y\right)^{1 / 2} .
$$

Now, using Hölder's inequality, we have

$$
\begin{aligned}
& \left(\int_{\mathbb{R}^{n}} \int_{K}\|x\|^{2 a}|f(x, k)|^{2} d k d x\right)^{\frac{1}{a}}\left(\int_{\mathbb{R}^{n}} \int_{K}|f(x, k)|^{2} d k d x\right)^{1-\frac{1}{a}} \\
& \geq \int_{\mathbb{R}^{n}} \int_{K}\|x\|^{2}|f(x, k)|^{\frac{2}{a}}|f(x, k)|^{2\left(1-\frac{1}{a}\right)} d k d x \\
& =\int_{\mathbb{R}^{n}} \int_{K}\|x\|^{2}|f(x, k)|^{2} d k d x,
\end{aligned}
$$

which implies

$$
\int_{\mathbb{R}^{n}} \int_{K}\|x\|^{2}|f(x, k)|^{2} d k d x \leq\left(\int_{\mathbb{R}^{n}} \int_{K}\|x\|^{2 a}|f(x, k)|^{2} d k d x\right)^{\frac{1}{a}}\left(\|f\|_{2}^{2}\right)^{1-\frac{1}{a}} .
$$

Combining (2.2) and (2.3), we obtain

$$
\begin{aligned}
\frac{n\|f\|_{2}^{2}}{4 \pi} \leq & \left(\int_{\mathbb{R}^{n}} \int_{K}\|x\|^{2 a}|f(x, k)|^{2} d k d x\right)^{\frac{1}{2 a}}\left(\|f\|_{2}^{2}\right)^{\frac{1}{2}-\frac{1}{2 a}} \\
& \times\left(\int_{\mathbb{R}^{n}}\|y\|^{2} \int_{A^{\prime}}\left|\hat{f}_{k}(y)\right|^{2} d k d y\right)^{1 / 2} .
\end{aligned}
$$


Since

$$
\int_{\mathbb{R}^{n}} \int_{A^{\prime}}\left|\mathscr{F}_{1} f(y, k)\right|^{2} d y d k=\int_{\mathbb{R}^{n}} \int_{A^{\prime}}|f(x, k)|^{2} d x d k=\|f\|_{2}^{2}<\infty
$$

therefore, $\mathscr{F}_{1} f \in L^{2}\left(\mathbb{R}^{n} \times A^{\prime}\right)$. Therefore, $\mathscr{F}_{2} \mathscr{F}_{1} f$ is well defined a.e. By approximating $f \in$ $L^{2}\left(\mathbb{R}^{n} \times A^{\prime}\right)$ by functions in $L^{1} \cap L^{2}\left(\mathbb{R}^{n} \times A^{\prime}\right)$, we have

$$
\mathscr{F}_{2} \mathscr{F}_{1} f=\hat{f}
$$

for all $f \in L^{2}\left(\mathbb{R}^{n} \times A^{\prime}\right)$. Applying the Plancherel formula on the locally compact group $K$, we have

$$
\int_{A^{\prime}}\left|\hat{f}_{k}(y)\right|^{2} d k=\int_{\widehat{K}}\|\hat{f}(y, \sigma)\|_{\mathrm{HS}}^{2} d \sigma
$$

Thus, (2.4) can be written as

$$
\begin{aligned}
\frac{n\|f\|_{2}^{2}}{4 \pi} \leq & \left(\int_{\mathbb{R}^{n}} \int_{K}\|x\|^{2 a}|f(x, k)|^{2} d k d x\right)^{\frac{1}{2 a}}\left(\|f\|_{2}^{2}\right)^{\frac{1}{2}-\frac{1}{2 a}} \\
& \times\left(\int_{\mathbb{R}^{n}} \int_{\widehat{K}}\|y\|^{2}\|\hat{f}(y, \sigma)\|_{\mathrm{HS}}^{2} d y d \sigma\right)^{1 / 2} .
\end{aligned}
$$

Now, again using Hölder's inequality, we have

$$
\begin{aligned}
& \left(\int_{\mathbb{R}^{n}} \int_{\widehat{K}}\|y\|^{2 b}\|\hat{f}(y, \sigma)\|_{\mathrm{HS}}^{2} d y d \sigma\right)^{\frac{1}{b}}\left(\int_{\mathbb{R}^{n}} \int_{\widehat{K}}\|\hat{f}(y, \sigma)\|_{\mathrm{HS}}^{2} d y d \sigma\right)^{1-\frac{1}{b}} \\
& \geq \int_{\mathbb{R}^{n}} \int_{\widehat{K}}\|y\|^{2}\|\hat{f}(y, \sigma)\|_{\mathrm{HS}}^{\frac{2}{b}}\|\hat{f}(y, \sigma)\|_{\mathrm{HS}}^{2\left(1-\frac{1}{b}\right)} d y d \sigma \\
& =\int_{\mathbb{R}^{n}} \int_{\widehat{K}}\|y\|^{2}\|\hat{f}(y, \sigma)\|_{\mathrm{HS}}^{2} d y d \sigma,
\end{aligned}
$$

which implies

$$
\int_{\mathbb{R}^{n}} \int_{\widehat{K}}\|y\|^{2}\|\hat{f}(y, \sigma)\|_{\mathrm{HS}}^{2} d y d \sigma \leq\left(\int_{\mathbb{R}^{n}} \int_{\widehat{K}}\|y\|^{2 b}\|\hat{f}(y, \sigma)\|_{\mathrm{HS}}^{2} d y d \sigma\right)^{\frac{1}{b}}\left(\|f\|_{2}^{2}\right)^{1-\frac{1}{b}}
$$

Combining (2.5) and (2.6), we obtain

$$
\begin{aligned}
\frac{n\|f\|_{2}^{2}}{4 \pi} \leq & \left(\int_{\mathbb{R}^{n}} \int_{K}\|x\|^{2 a}|f(x, k)|^{2} d k d x\right)^{\frac{1}{2 a}}\left(\|f\|_{2}^{2}\right)^{\frac{1}{2}-\frac{1}{2 a}} \\
& \times\left(\int_{\mathbb{R}^{n}} \int_{\widehat{K}}\|y\|^{2 b}\|\hat{f}(y, \sigma)\|_{\mathrm{HS}}^{2} d y d \sigma\right)^{\frac{1}{2 b}}\left(\|f\|_{2}^{2}\right)^{\frac{1}{2}-\frac{1}{2 b}},
\end{aligned}
$$

which implies

$$
\frac{n\|f\|_{2}^{\left(\frac{1}{a}+\frac{1}{b}\right)}}{4 \pi} \leq\left(\int_{\mathbb{R}^{n}} \int_{K}\|x\|^{2 a}|f(x, k)|^{2} d k d x\right)^{\frac{1}{2 a}}\left(\int_{\mathbb{R}^{n}} \int_{\widehat{K}}\|y\|^{2 b}\|\hat{f}(y, \sigma)\|_{\mathrm{HS}}^{2} d y d \sigma\right)^{\frac{1}{2 b}} .
$$




\section{Euclidean motion group $M(n)$}

Consider $M(n)$ to be the semi-direct product of $\mathbb{R}^{n}$ with $K=\mathrm{SO}(n)$. The group law is given by

$$
(z, k)\left(w, k^{\prime}\right)=\left(z+k \cdot w, k k^{\prime}\right)
$$

for $z, w \in \mathbb{R}^{n}$ and $k, k^{\prime} \in K$. The group $M(n)$ is called the motion group of the Euclidean plane $\mathbb{R}^{n}$.

As in [5], $M=\mathrm{SO}(n-1)$ can be considered as a subgroup of $K$ leaving the point $e_{1}=$ $(1,0,0, \ldots, 0)$ fixed. All the irreducible unitary representations of $M(n)$ relevant for the Plancherel formula are parametrized (up to unitary equivalence) by pairs $(\lambda, \sigma)$, where $\lambda>0$ and $\sigma \in \widehat{M}$, the unitary dual of $M$.

Given $\sigma \in \widehat{M}$ realized on a Hilbert space $H_{\sigma}$ of dimension $d_{\sigma}$, consider the space,

$$
\begin{array}{r}
L^{2}(K, \sigma)=\left\{\varphi \mid \varphi: K \rightarrow M_{d_{\sigma} \times d_{\sigma}}, \int\|\varphi(k)\|^{2} d k<\infty,\right. \\
\varphi(u k)=\sigma(u) \varphi(k), \text { for } u \in M \text { and } k \in K\} .
\end{array}
$$

Note that $L^{2}(K, \sigma)$ is a Hilbert space under the inner product

$$
\langle\varphi, \psi\rangle=\int_{K} \operatorname{tr}\left(\varphi(k) \psi(k)^{*}\right) d k
$$

For each $\lambda>0$ and $\sigma \in \widehat{M}$, we can define a representation $\pi_{\lambda, \sigma}$ of $M(n)$ on $L^{2}(K, \sigma)$ as follows.

$$
\text { For } \varphi \in L^{2}(K, \sigma),(z, k) \in M(n) \text {, }
$$

$$
\pi_{\lambda, \sigma}(z, k) \varphi(u)=e^{i \lambda\left\langle u^{-1} \cdot e_{1}, z\right\rangle} \varphi(u k)
$$

for $u \in K$.

If $\varphi_{j}(k)$ are the column vectors of $\varphi \in L^{2}(K, \sigma)$, then $\varphi_{j}(u k)=\sigma(u) \varphi_{j}(k)$ for all $u \in M$. Therefore, $L^{2}(K, \sigma)$ can be written as the direct sum of $d_{\sigma}$ copies of $H(K, \sigma)$, where

$$
\begin{aligned}
H(K, \sigma)= & \left\{\varphi \mid \varphi: K \rightarrow \mathbb{C}^{d_{\sigma}}, \int\|\varphi(k)\|^{2} d k<\infty,\right. \\
& \varphi(u k)=\sigma(u) \varphi(k), \text { for } u \in M \text { and } k \in K\} .
\end{aligned}
$$

It can be shown that $\pi_{\lambda, \sigma}$ restricted to $H(K, \sigma)$ is an irreducible unitary representation of $M(n)$. Moreover, any irreducible unitary representation of $M(n)$ which is infinite dimensional is unitarily equivalent to one and only one $\pi_{\lambda, \sigma}$.

The Fourier transform of $f \in L^{2}(M(n))$ is given by

$$
\hat{f}(\lambda, \sigma)=\int_{M(n)} f(z, k) \pi_{\lambda, \sigma}(z, k)^{*} d z d k
$$

$\hat{f}(\lambda, \sigma)$ is a Hilbert-Schmidt operator on $H(K, \sigma)$. 
A solid harmonic of degree $m$ is a polynomial which is homogeneous of degree $m$ and whose Laplacian is zero. The set of all such polynomials will be denoted by $\mathbb{H}_{m}$ and the restriction of elements of $\mathbb{H}_{m}$ to $S^{n-1}$ is denoted by $S_{m}$. By choosing an orthonormal basis $\left\{g_{m j}: j=1,2, \ldots, d_{m}\right\}$ of $S_{m}$ for each $m=0,1,2, \ldots$, we get an orthonormal basis for $L^{2}\left(S^{n-1}\right)$.

The Haar measure on $M(n)$ is $d g=d z d k$, where $d z$ is Lebesgue measure on $\mathbb{R}^{n}$ and $d k$ is the normalized Haar measure on $\mathrm{SO}(n)$.

The Plancherel formula on $M(n)$ is given as follows (see [6]).

Proposition 3.1 (Plancherel formula) Let $f \in L^{2}(M(n))$, then

$$
\int_{M(n)}\left|f\left(z_{1}, z_{2}, \ldots, z_{n}, k\right)\right|^{2} d z_{1} d z_{2} \cdots d z_{n} d k=c_{n} \int_{0}^{\infty}\left(\sum_{\sigma \in \widehat{M}} d_{\sigma}\|\hat{f}(\lambda, \sigma)\|_{\mathrm{HS}}^{2}\right) \lambda^{n-1} d \lambda,
$$

where $c_{n}=\frac{2}{2^{n / 2} \Gamma\left(\frac{n}{2}\right)}$.

We shall now state and prove the following generalized Heisenberg uncertainty inequality for a Fourier transform on $M(n)$.

Theorem 3.2 For any $f \in L^{2}(M(n))$ and $a, b \geq 1$, we have

$$
\begin{aligned}
\frac{\|f\|_{2}^{\left(\frac{1}{a}+\frac{1}{b}\right)}}{2 \sqrt{c_{n}}} \leq & \left(\int_{K} \int_{\mathbb{R}^{n}}\|z\|^{2 a}|f(z, k)|^{2} d z d k\right)^{\frac{1}{2 a}} \\
& \times\left(\int_{0}^{\infty} \sum_{\sigma \in \widehat{M}} d_{\sigma} \lambda^{2 b}\|\hat{f}(\lambda, \sigma)\|_{\mathrm{HS}}^{2} \lambda^{n-1} d \lambda\right)^{\frac{1}{2 b}} .
\end{aligned}
$$

Proof Consider the norm $\|\cdot\|$ on $L^{2}(M(n))$ defined by

$$
\begin{aligned}
\|f\|:= & \left(\int_{\mathbb{R}^{n}} \int_{K}\left(1+\|z\|^{2 a}\right)|f(z, k)|^{2} d z d k\right)^{1 / 2} \\
& +\left(\int_{0}^{\infty} \sum_{\sigma \in \widehat{M}} d_{\sigma}\left(1+\lambda^{2 b}\right)\|\hat{f}(\lambda, \sigma)\|_{\mathrm{HS}}^{2} \lambda^{n-1} d \lambda\right)^{1 / 2} .
\end{aligned}
$$

This gives us a Banach space $B=\left\{f \in L^{2}(G):\|f\|<\infty\right\}$, which is contained in $L^{2}(M(n))$ and the space $\mathcal{S}(M(n))$ of $C^{\infty}$-functions which are rapidly decreasing on $M(n)$ can be shown to be dense in $B$. It suffices to prove the inequality of Theorem 3.2 for functions in $\mathcal{S}(M(n))$; it is automatically valid for any $f \in B$. If $0 \neq f \in L^{2}(M(n)) \backslash B$, then the right-hand side of the inequality is always $+\infty$ and the inequality is trivially valid.

Let $f \in \mathcal{S}(M(n))$. Assuming that both integrals on the right-hand side of (3.1) are finite, we have

$$
\int_{\mathbb{R}^{n}}|f(z, k)|^{2} d z<\infty, \quad \text { for all } k \in K
$$

For $k \in K$, we define $f_{k}(z)=f(z, k)$, for every $z \in \mathbb{R}^{n}$.

Clearly, $f_{k} \in L^{2}\left(\mathbb{R}^{n}\right)$, for all $k \in K$.

Take $z=\left(z_{1}, z_{2}, \ldots, z_{n}\right)$ and $w=\left(w_{1}, w_{2}, \ldots, w_{n}\right)$. 
Bansal and Kumar Journal of Inequalities and Applications （2015) 2015:168

Page 7 of 15

By the Heisenberg inequality on $\mathbb{R}^{n}$, we have

$$
\begin{aligned}
& \frac{\left\|f_{k}\right\|_{2}^{2}}{4 \pi} \leq\left(\int_{\mathbb{R}^{n}}\left|z_{1}\right|^{2}\left|f_{k}(z)\right|^{2} d z\right)^{1 / 2}\left(\int_{\mathbb{R}^{n}}\left|w_{1}\right|^{2}\left|\hat{f}_{k}(w)\right|^{2} d w\right)^{1 / 2} \\
& \Rightarrow \frac{1}{4 \pi} \int_{\mathbb{R}^{n}}|f(z, k)|^{2} d z \leq\left(\int_{\mathbb{R}^{n}}\left|z_{1}\right|^{2}|f(z, k)|^{2} d z\right)^{1 / 2}\left(\int_{\mathbb{R}^{n}}\left|w_{1}\right|^{2}\left|\hat{f}_{k}(w)\right|^{2} d w\right)^{1 / 2} .
\end{aligned}
$$

Integrating both sides with respect to $d k$, we get

$$
\frac{1}{4 \pi} \int_{K} \int_{\mathbb{R}^{n}}|f(z, k)|^{2} d z d k \leq \int_{K}\left(\int_{\mathbb{R}^{n}}\left|z_{1}\right|^{2}|f(z, k)|^{2} d z\right)^{1 / 2}\left(\int_{\mathbb{R}^{n}}\left|w_{1}\right|^{2}\left|\hat{f}_{k}(w)\right|^{2} d w\right)^{1 / 2} d k
$$

which implies

$$
\begin{aligned}
\frac{\|f\|_{2}^{2}}{4 \pi} & \leq \int_{K}\left(\int_{\mathbb{R}^{n}}\left|z_{1}\right|^{2}|f(z, k)|^{2} d z\right)^{1 / 2}\left(\int_{\mathbb{R}^{n}}\left|w_{1}\right|^{2}\left|\hat{f}_{k}(w)\right|^{2} d w\right)^{1 / 2} d k \\
& \leq\left(\int_{K} \int_{\mathbb{R}^{n}}\left|z_{1}\right|^{2}|f(z, k)|^{2} d z d k\right)^{1 / 2}\left(\int_{K} \int_{\mathbb{R}^{n}}\left|w_{1}\right|^{2}\left|\hat{f}_{k}(w)\right|^{2} d w d k\right)^{1 / 2}
\end{aligned}
$$

(by the Cauchy-Schwarz inequality)

$$
\leq\left(\int_{K} \int_{\mathbb{R}^{n}}\|z\|^{2}|f(z, k)|^{2} d z d k\right)^{1 / 2}\left(\int_{K} \int_{\mathbb{R}^{n}}\left|w_{1}\right|^{2}\left|\hat{f}_{k}(w)\right|^{2} d w d k\right)^{1 / 2} .
$$

Now,

$$
\begin{aligned}
& \left(\int_{K} \int_{\mathbb{R}^{n}}\|z\|^{2 a}|f(z, k)|^{2} d z d k\right)^{\frac{1}{a}}\left(\int_{K} \int_{\mathbb{R}^{n}}|f(z, k)|^{2} d z d k\right)^{1-\frac{1}{a}} \\
& \quad=\left(\int_{K} \int_{\mathbb{R}^{n}}\left(\|z\|^{2}|f(z, k)|^{\frac{2}{a}}\right)^{a} d z d k\right)^{\frac{1}{a}}\left(\int_{K} \int_{\mathbb{R}^{n}}\left(|f(z, k)|^{2\left(1-\frac{1}{a}\right)}\right)^{\frac{1}{\left(1-\frac{1}{a}\right)}} d z d k\right)^{1-\frac{1}{a}} \\
& \quad \geq \int_{K} \int_{\mathbb{R}^{n}}\|z\|^{2}|f(z, k)|^{\frac{2}{a}}|f(z, k)|^{2\left(1-\frac{1}{a}\right)} d z d k \quad \text { (by Hölder's inequality) } \\
& \quad=\int_{K} \int_{\mathbb{R}^{n}}\|z\|^{2}|f(z, k)|^{2} d z d k .
\end{aligned}
$$

Combining (3.2) and (3.3), we get

$$
\begin{aligned}
\frac{\|f\|_{2}^{2}}{4 \pi} \leq & \left(\int_{K} \int_{\mathbb{R}^{n}}\|z\|^{2 a}|f(z, k)|^{2} d z d k\right)^{\frac{1}{2 a}}\left(\|f\|_{2}^{2}\right)^{\frac{1}{2}-\frac{1}{2 a}} \\
& \times\left(\int_{K} \int_{\mathbb{R}^{n}}\left|w_{1}\right|^{2}\left|\hat{f}_{k}(w)\right|^{2} d w d k\right)^{1 / 2} .
\end{aligned}
$$

Now, using the Plancherel formula on $\mathbb{R}^{n}$, we have

$$
\begin{aligned}
& \int_{K} \int_{\mathbb{R}^{n}}\left|w_{1}\right|^{2}\left|\hat{f}_{k}(w)\right|^{2} d w d k \\
& \quad=\int_{K} \int_{\mathbb{R}^{n}}\left|w_{1}\right|^{2}\left|\int_{\mathbb{R}^{n}} f(z, k) e^{-2 \pi i\langle z, w\rangle} d z\right|^{2} d w d k
\end{aligned}
$$




$$
\begin{aligned}
& =\int_{K} \int_{\mathbb{R}^{n}}\left|w_{1}\right|^{2}\left|\mathscr{F}_{1,2, \ldots, n} f\left(w_{1}, w_{2}, \ldots, w_{n}, k\right)\right|^{2} d w_{1} d w_{2} \cdots d w_{n} d k \\
& =\int_{K} \int_{\mathbb{R}^{n}}\left|w_{1}\right|^{2}\left|\mathscr{F}_{1} f\left(w_{1}, z_{2}, \ldots, z_{n}, k\right)\right|^{2} d w_{1} d z_{2} \cdots d z_{n} d k .
\end{aligned}
$$

Since $\frac{\partial f}{\partial z_{1}} \in \mathcal{S}(M(n))$, we have

$$
\int_{\mathbb{R}}\left|\frac{\partial f}{\partial z_{1}}\left(z_{1}, z_{2}, \ldots, z_{n}, k\right)\right|^{2} d z_{1}<\infty
$$

for all $z_{i} \in \mathbb{R}$ and $k \in K$.

Therefore, $w_{1} \mathscr{F}_{1} f\left(w_{1}, z_{2}, \ldots, z_{n}, k\right) \in L^{2}(\mathbb{R})$ and

$$
\left(\frac{\partial f}{\partial z_{1}}\left(z_{1}, z_{2}, \ldots, z_{n}, k\right)\right)^{\wedge}\left(w_{1}\right)=2 \pi i w_{1} \mathscr{F}_{1} f\left(w_{1}, z_{2}, \ldots, z_{n}, k\right)
$$

for all $z_{i} \in \mathbb{R}$ and $k \in K$. Then

$$
\begin{gathered}
\int_{\mathbb{R}}\left|w_{1}\right|^{2}\left|\mathscr{F}_{1} f\left(w_{1}, z_{2}, \ldots, z_{n}, k\right)\right|^{2} d w_{1} \\
\quad=\frac{1}{4 \pi^{2}} \int_{\mathbb{R}}\left|\frac{\partial f}{\partial z_{1}}\left(z_{1}, z_{2}, \ldots, z_{n}, k\right)\right|^{2} d z_{1},
\end{gathered}
$$

which implies

$$
\begin{aligned}
& \int_{K} \int_{\mathbb{R}^{n}}\left|w_{1}\right|^{2}\left|\mathscr{F}_{1} f\left(w_{1}, z_{2}, \ldots, z_{n}, k\right)\right|^{2} d w_{1} d z_{2} \cdots d z_{n} d k \\
& \quad=\frac{1}{4 \pi^{2}} \int_{K} \int_{\mathbb{R}^{n}}\left|\frac{\partial f}{\partial z_{1}}\left(z_{1}, z_{2}, \ldots, z_{n}, k\right)\right|^{2} d z_{1} d z_{2} \cdots d z_{n} d k .
\end{aligned}
$$

By Proposition 3.1, we obtain

$$
\begin{gathered}
\int_{K} \int_{\mathbb{R}^{n}}\left|\frac{\partial f}{\partial z_{1}}\left(z_{1}, z_{2}, \ldots, z_{n}, k\right)\right|^{2} d z_{1} d z_{2} \cdots d z_{n} d k \\
\quad=c_{n} \int_{0}^{\infty} \sum_{\sigma \in \widehat{M}} d_{\sigma}\left\|\left(\frac{\partial f}{\partial z_{1}}\right)^{\wedge}(\lambda, \sigma)\right\|_{\mathrm{HS}}^{2} \lambda^{n-1} d \lambda
\end{gathered}
$$

Combining (3.4), (3.5), (3.6), and (3.7), we obtain

$$
\begin{aligned}
\frac{\|f\|_{2}^{2}}{2 \sqrt{c_{n}}} \leq & \left(\int_{K} \int_{\mathbb{R}^{n}}\|z\|^{2 a}|f(z, k)|^{2} d z d k\right)^{\frac{1}{2 a}}\left(\|f\|_{2}^{2}\right)^{\frac{1}{2}-\frac{1}{2 a}} \\
& \times\left(\int_{0}^{\infty} \sum_{\sigma \in \widehat{M}} d_{\sigma}\left\|\left(\frac{\partial f}{\partial z_{1}}\right)^{\wedge}(\lambda, \sigma)\right\|_{\mathrm{HS}}^{2} \lambda^{n-1} d \lambda\right)^{1 / 2} .
\end{aligned}
$$

For each $\lambda>0$ and $\sigma \in \widehat{M}$, consider the representation $\pi_{\lambda, \sigma}(z, k)$ realized on $L^{2}(K, \sigma)$ as

$$
\pi_{\lambda, \sigma}(z, k) g(u)=e^{i \lambda\left\langle u^{-1} \cdot e_{1}, z\right\rangle} g(u k), \quad u \in \mathrm{SO}(n)
$$


Denote $u=\left[u_{i j}\right]_{n \times n}$; we have

$$
u^{-1} \cdot e_{1}=u^{T} \cdot e_{1}=\left[\begin{array}{llll}
u_{11} & u_{12} & \cdots & u_{1 n}
\end{array}\right]^{T} .
$$

Therefore, $\left\langle u^{-1} \cdot e_{1}, z\right\rangle=\sum_{i=1}^{n} u_{1 i} z_{i}$.

Since $f \in \mathcal{S}(M(n))$,

$$
\begin{aligned}
& \left(\frac{\partial f}{\partial z_{1}}\right)^{\wedge}(\lambda, \sigma) g(u) \\
& =\int_{\mathbb{R}^{n}} \int_{K} \frac{\partial f}{\partial z_{1}}\left(z_{1}, z_{2}, \ldots, z_{n}, k\right) \pi_{\lambda, \sigma}\left(z_{1}, z_{2}, \ldots, z_{n}, k\right)^{*} g(u) d z_{1} d z_{2} \cdots d z_{n} d k \\
& =\int_{\mathbb{R}^{n}} \int_{K} \lim _{h \rightarrow 0}\left[\frac{f\left(z_{1}+h, z_{2}, \ldots, z_{n}, k\right)-f\left(z_{1}, z_{2}, \ldots, z_{n}, k\right)}{h}\right] \\
& \times \pi_{\lambda, \sigma}\left(z_{1}, z_{2}, \ldots, z_{n}, k\right)^{*} g(u) d z_{1} d z_{2} \ldots d z_{n} d k \\
& =\lim _{h \rightarrow 0} \frac{1}{h}\left[\int_{\mathbb{R}^{n}} \int_{K} f\left(z_{1}+h, z_{2}, \ldots, z_{n}, k\right) \pi_{\lambda, \sigma}\left(z_{1}, z_{2}, \ldots, z_{n}, k\right)^{*} g(u) d z_{1} d z_{2} \cdots d z_{n} d k\right. \\
& \left.-\int_{\mathbb{R}^{n}} \int_{K} f\left(z_{1}, z_{2}, \ldots, z_{n}, k\right) \pi_{\lambda, \sigma}\left(z_{1}, z_{2}, \ldots, z_{n}, k\right)^{*} g(u) d z_{1} d z_{2} \cdots d z_{n} d k\right] \\
& =\lim _{h \rightarrow 0} \frac{1}{h}\left[\int_{\mathbb{R}^{n}} \int_{K} f\left(z_{1}, z_{2}, \ldots, z_{n}, k\right) e^{-i \lambda h u_{11}} \pi_{\lambda, \sigma}\left(z_{1}, z_{2}, \ldots, z_{n}, k\right)^{*}\right. \\
& \times g(u) d z_{1} d z_{2} \cdots d z_{n} d k \\
& \left.-\int_{\mathbb{R}^{n}} \int_{K} f\left(z_{1}, z_{2}, \ldots, z_{n}, k\right) \pi_{\lambda, \sigma}\left(z_{1}, z_{2}, \ldots, z_{n}, k\right)^{*} g(u) d z_{1} d z_{2} \cdots d z_{n} d k\right] \\
& =\lim _{h \rightarrow 0}\left[\frac{e^{-i \lambda h u_{11}}-1}{h}\right] \int_{\mathbb{R}^{n}} \int_{K} f\left(z_{1}, z_{2}, \ldots, z_{n}, k\right) \pi_{\lambda, \sigma}\left(z_{1}, z_{2}, \ldots, z_{n}, k\right)^{*} \\
& \times g(u) d z_{1} d z_{2} \cdots d z_{n} d k \\
& =i \lambda u_{11} \int_{\mathbb{R}^{n}} \int_{K} f\left(z_{1}, z_{2}, \ldots, z_{n}, k\right) \pi_{\lambda, \sigma}\left(z_{1}, z_{2}, \ldots, z_{n}, k\right)^{*} g(u) d z_{1} d z_{2} \cdots d z_{n} d k \\
& =i \lambda u_{11} \hat{f}(\lambda, \sigma) g(u)
\end{aligned}
$$

Hence,

$$
\begin{aligned}
\left\|\left(\frac{\partial f}{\partial z_{1}}\right)^{\wedge}(\lambda, \sigma)\right\|_{\mathrm{HS}}^{2} & =\sum_{m=0}^{\infty} \sum_{j=1}^{d_{m}} \int_{K}\left|i \lambda u_{11} \hat{f}(\lambda, \sigma) g_{m j}(u)\right|^{2} d u \\
& \leq \lambda^{2} \sum_{m=0}^{\infty} \sum_{j=1}^{d_{m}} \int_{K}\left|\hat{f}(\lambda, \sigma) g_{m j}(u)\right|^{2} d u=\lambda^{2}\|\hat{f}(\lambda, \sigma)\|_{\mathrm{HS}}^{2}
\end{aligned}
$$

Therefore, (3.8) can be written as

$$
\begin{aligned}
\frac{\|f\|_{2}^{2}}{2 \sqrt{c_{n}}} \leq & \left(\int_{K} \int_{\mathbb{R}^{n}}\|z\|^{2 a}|f(z, k)|^{2} d z d k\right)^{\frac{1}{2 a}}\left(\|f\|_{2}^{2}\right)^{\frac{1}{2}-\frac{1}{2 a}} \\
& \times\left(\int_{0}^{\infty} \sum_{\sigma \in \widehat{M}} d_{\sigma} \lambda^{2}\|\hat{f}(\lambda, \sigma)\|_{\mathrm{HS}}^{2} \lambda^{n-1} d \lambda\right)^{1 / 2} .
\end{aligned}
$$


Now, again using Hölder's inequality, we have

$$
\begin{aligned}
& \left(\int_{0}^{\infty} \sum_{\sigma \in \widehat{M}} d_{\sigma} \lambda^{2 b}\|\hat{f}(\lambda, \sigma)\|_{\mathrm{HS}}^{2} \lambda^{n-1} d \lambda\right)^{\frac{1}{b}}\left(\int_{0}^{\infty} \sum_{\sigma \in \widehat{M}} d_{\sigma}\|\hat{f}(\lambda, \sigma)\|_{\mathrm{HS}}^{2} \lambda^{n-1} d \lambda\right)^{1-\frac{1}{b}} \\
& \geq \int_{0}^{\infty} \sum_{\sigma \in \widehat{M}} d_{\sigma}^{1 / b} \lambda^{2}\|\hat{f}(\lambda, \sigma)\|_{\mathrm{HS}}^{\frac{2}{b}} d_{\sigma}^{\left(1-\frac{1}{b}\right)}\|\hat{f}(\lambda, \sigma)\|_{\mathrm{HS}}^{2\left(1-\frac{1}{b}\right)} \lambda^{n-1} d \lambda \\
& =\int_{0}^{\infty} \sum_{\sigma \in \widehat{M}} d_{\sigma} \lambda^{2}\|\hat{f}(\lambda, \sigma)\|_{\mathrm{HS}}^{2} \lambda^{n-1} d \lambda
\end{aligned}
$$

which implies

$$
\begin{aligned}
& \int_{0}^{\infty} \sum_{\sigma \in \widehat{M}} d_{\sigma} \lambda^{2}\|\hat{f}(\lambda, \sigma)\|_{\mathrm{HS}}^{2} \lambda^{n-1} d \lambda \\
& \quad \leq\left(\int_{0}^{\infty} \sum_{\sigma \in \widehat{M}} d_{\sigma} \lambda^{2 b}\|\hat{f}(\lambda, \sigma)\|_{\mathrm{HS}}^{2} \lambda^{n-1} d \lambda\right)^{\frac{1}{b}}\left(\|f\|_{2}^{2}\right)^{1-\frac{1}{b}}
\end{aligned}
$$

Combining (3.9) and (3.10), we obtain

$$
\begin{aligned}
\frac{\|f\|_{2}^{\left(\frac{1}{a}+\frac{1}{b}\right)}}{2 \sqrt{c_{n}}} \leq & \left(\int_{K} \int_{\mathbb{R}^{n}}\|z\|^{2 a}|f(z, k)|^{2} d z d k\right)^{\frac{1}{2 a}} \\
& \times\left(\int_{0}^{\infty} \sum_{\sigma \in \widehat{M}} d_{\sigma} \lambda^{2 b}\|\hat{f}(\lambda, \sigma)\|_{\mathrm{HS}}^{2} \lambda^{n-1} d \lambda\right)^{\frac{1}{2 b}} .
\end{aligned}
$$

\section{A class of nilpotent Lie groups}

In this section, we shall prove the Heisenberg uncertainty inequality for a class of connected, simply connected nilpotent Lie groups $G$ for which the Hilbert-Schmidt norm of the group Fourier transform $\pi_{\xi}(f)$ of $f$ attains a particular form.

Let $\mathfrak{g}$ be an $n$-dimensional real nilpotent Lie algebra, and let $G=\exp \mathfrak{g}$ be the associated connected and simply connected nilpotent Lie group [7]. Let $\mathcal{B}=\left\{X_{1}, X_{2}, \ldots, X_{n}\right\}$ be a strong Malcev basis of $\mathfrak{g}$ through the ascending central series of $\mathfrak{g}$. We introduce a 'norm function' on $G$ by setting, for $x=\exp \left(x_{1} X_{1}+x_{2} X_{2}+\cdots+x_{n} X_{n}\right) \in G, x_{j} \in \mathbb{R}$,

$$
\|x\|=\left(x_{1}^{2}+\cdots+x_{n}^{2}\right)^{1 / 2} \text {. }
$$

The composed map

$$
\mathbb{R}^{n} \rightarrow \mathfrak{g} \rightarrow G
$$

given as

$$
\left(x_{1}, \ldots, x_{n}\right) \rightarrow \sum_{j=1}^{n} x_{j} X_{j} \rightarrow \exp \left(\sum_{j=1}^{n} x_{j} X_{j}\right)
$$

is a diffeomorphism and maps a Lebesgue measure on $\mathbb{R}^{n}$ to a Haar measure on $G$. In this manner, we shall always identify $\mathfrak{g}$, and sometimes $G$, as sets with $\mathbb{R}^{n}$. Thus, measurable (integrable) functions on $G$ can be viewed as such functions on $\mathbb{R}^{n}$. 
Let $\mathfrak{g}^{*}$ denote the vector space dual of $\mathfrak{g}$ and $\left\{X_{1}^{*}, \ldots, X_{n}^{*}\right\}$ the basis of $\mathfrak{g}^{*}$ which is dual to $\left\{X_{1}, \ldots, X_{n}\right\}$. Then $\left\{X_{1}^{*}, \ldots, X_{n}^{*}\right\}$ is a Jordan-Hölder basis for the coadjoint action of $G$ on $\mathfrak{g}^{*}$. We shall identify $\mathfrak{g}^{*}$ with $\mathbb{R}^{n}$ via the map

$$
\xi=\left(\xi_{1}, \ldots, \xi_{n}\right) \rightarrow \sum_{j=1}^{n} \xi_{j} X_{j}^{*}
$$

and on $\mathfrak{g}^{*}$ we introduce the Euclidean norm relative to the basis $\left\{X_{1}^{*}, \ldots, X_{n}^{*}\right\}$, i.e.

$$
\left\|\sum_{j=1}^{n} \xi_{j} X_{j}^{*}\right\|=\left(\xi_{1}^{2}+\cdots+\xi_{n}^{2}\right)=\|\xi\| .
$$

Let $\mathfrak{g}_{j}=\mathbb{R}-\operatorname{span}\left\{X_{1}, \ldots, X_{n}\right\}$. For $\xi \in \mathfrak{g}^{*}, \mathcal{O}_{\xi}$ denotes the coadjoint orbit of $\xi$. An index $j \in$ $\{1,2, \ldots, n\}$ is a jump index for $\xi$ if

$$
\mathfrak{g}(\xi)+\mathfrak{g}_{j} \neq \mathfrak{g}(\xi)+\mathfrak{g}_{j-1}
$$

We consider

$$
e(\xi)=\{j: j \text { is a jump index for } \xi\}
$$

This set contains exactly $\operatorname{dim}\left(\mathcal{O}_{l}\right)$ indices. Also, there are two disjoint sets $S$ and $T$ of indices with $S \cup T=\{1, \ldots, n\}$ and a $G$-invariant Zariski open set $\mathcal{U}$ of $\mathfrak{g}^{*}$ such that $e(\xi)=S$ for all $\xi \in \mathcal{U}$. We define the Pfaffian $\operatorname{Pf}(\xi)$ of the skew-symmetric matrix $M_{S}(\xi)=$ $\left(\xi\left(\left[X_{i}, X_{j}\right]\right)\right)_{i, j \in S}$ as

$$
|\operatorname{Pf}(\xi)|^{2}=\operatorname{det} M_{S}(\xi)
$$

Let $V_{S}=\mathbb{R}$-span $\left\{X_{i}^{*}: i \in S\right\}, V_{T}=\mathbb{R}$-span $\left\{X_{i}^{*}: i \in T\right\}$, and $d \xi$ be the Lebesgue measure on $V_{T}$ such that the unit cube spanned by $\left\{X_{i}^{*}: i \in T\right\}$ has volume 1 . Then $\mathfrak{g}^{*}=V_{T} \oplus V_{S}$ and $V_{T}$ meets $\mathcal{U}$. Let $\mathcal{W}=\mathcal{U} \cap V_{T}$ be the cross section for the coadjoint orbits through the points in $\mathcal{U}$. If $d \xi$ is the Lebesgue measure on $\mathcal{W}$, then $d \mu(\xi)=|\operatorname{Pf}(\xi)| d \xi$ is a Plancherel measure for $\widehat{G}$. The Plancherel formula is given by

$$
\|f\|_{2}^{2}=\int_{\mathcal{W}}\left\|\pi_{\xi}(f)\right\|_{\mathrm{HS}}^{2} d \mu(\xi), \quad f \in L^{1} \cap L^{2}(G)
$$

where $\left\|\pi_{\xi}(f)\right\|_{H S}$ denotes the Hilbert-Schmidt norm of $\pi_{\xi}(f)$ and $d g$ is the Haar measure on $G$.

We shall consider the case in which $\mathcal{W}$ takes the following form:

$$
\mathcal{W}=\left\{\xi=\left(\xi_{1}, \xi_{2}, \ldots, \xi_{n}\right) \in \mathfrak{g}^{*}: \xi_{j}=0 \text {, for }(n-k) \text { values of } j \text { with }|\operatorname{Pf}(\xi)| \neq 0\right\}
$$

We denote the vanishing variables by $\xi_{j_{1}}, \xi_{j_{2}}, \ldots, \xi_{j_{n-k}}$.

We consider the class of groups for which for all $\xi \in \mathcal{W}$ and $f \in L^{2}(G)$ the HilbertSchmidt norm $\left\|\pi_{\xi}(f)\right\|_{\mathrm{HS}}^{2}$ has the following form:

$$
\left\|\pi_{\xi}(f)\right\|_{\mathrm{HS}}^{2}=|h(\xi)| \int_{\mathbb{R}^{n-k}}\left|\mathscr{F}(f \circ \exp )\left(\xi_{1}, \xi_{2}+Q_{2}, \ldots, \xi_{n}+Q_{n}\right)\right|^{2} d \xi_{j_{1}} d \xi_{j_{2}} \cdots d \xi_{j_{n-k}}
$$


where $\mathscr{F}$ denotes the Fourier transform on $\mathbb{R}^{n-k} ; h$ is a function from $\mathcal{W}$ to $\mathbb{R}$ which is nonzero on $\mathcal{W}$ and the functions $Q_{m}=Q_{m}\left(\xi_{1}, \xi_{2}, \ldots, \xi_{m-1}\right)$ with $2 \leq m \leq n$.

We have the following Heisenberg uncertainty inequality for such groups.

Theorem 4.1 For any $f \in L^{1} \cap L^{2}(G)$ and $a, b \geq 1$, we have

$$
\begin{aligned}
\frac{\|f\|_{2}^{\left(\frac{1}{a}+\frac{1}{b}\right)}}{4 \pi} \leq & \left(\int_{G}\|x\|^{2 a}|f(x)|^{2} d x\right)^{\frac{1}{2 a}} \\
& \times\left(\int_{\mathcal{W}}\|\xi\|^{2 b}\left\|\pi_{\xi}(f)\right\|_{\mathrm{HS}}^{2} \frac{1}{|h(\xi)|^{b}|\operatorname{Pf}(\xi)|^{b-1}} d \xi\right)^{\frac{1}{2 b}} .
\end{aligned}
$$

Proof Assuming both integrals on the right-hand side of (4.1) to be finite, we have

$$
\begin{aligned}
& \left(\int_{G}\|x\|^{2}|f(x)|^{2} d x\right)^{1 / 2}\left(\int_{\mathcal{W}}\|\xi\|^{2}\left\|\pi_{\xi}(f)\right\|_{\mathrm{HS}}^{2} \frac{1}{|h(\xi)|} d \xi\right)^{1 / 2} \\
& =\left(\int_{\mathbb{R}^{n}} \sum_{i=1}^{n}\left|x_{i}\right|^{2}\left|(f \circ \exp )\left(\sum_{i=1}^{n} x_{i} X_{i}\right)\right|^{2} d x_{1} \cdots d x_{n}\right)^{1 / 2} \\
& \quad \times\left(\int_{\mathbb{R}^{k}} \int_{\mathbb{R}^{n-k}} \sum_{i=1}^{n}\left|\xi_{i}\right|^{2}\left|\mathscr{F}(f \circ \exp )\left(\xi_{1}, \xi_{2}+Q_{2}, \ldots, \xi_{n}+Q_{n}\right)\right|^{2} d \xi_{1} \cdots d \xi_{n}\right)^{1 / 2} \\
& \geq\left(\int_{\mathbb{R}^{n}}\left|x_{1}\right|^{2}\left|(f \circ \exp )\left(\sum_{i=1}^{n} x_{i} X_{i}\right)\right|^{2} d x_{1} \cdots d x_{n}\right)^{1 / 2} \\
& \quad \times\left(\int_{\mathbb{R}^{k}} \int_{\mathbb{R}^{n-k}}\left|\xi_{1}\right|^{2}\left|\mathscr{F}(f \circ \exp )\left(\xi_{1}, \xi_{2}+Q_{2}, \ldots, \xi_{n}+Q_{n}\right)\right|^{2} d \xi_{1} \cdots d \xi_{n}\right)^{1 / 2} \\
& =\left(\int_{\mathbb{R}^{n}}\left|x_{1}\right|^{2}\left|F\left(x_{1}, \ldots, x_{n}\right)\right|^{2} d x_{1} \cdots d x_{n}\right)^{1 / 2} \\
& \quad \times\left(\int_{\mathbb{R}^{n}}\left|\xi_{1}\right|^{2}\left|\widehat{F}\left(\xi_{1}, \xi_{2}, \ldots, \xi_{n}\right)\right|^{2} d \xi_{1} d \xi_{2} \cdots d \xi_{n}\right)^{1 / 2},
\end{aligned}
$$

where $F\left(x_{1}, \ldots, x_{n}\right)=(f \circ \exp )\left(\sum_{i=1}^{n} x_{i} X_{i}\right)$ which is in $L^{2}\left(R^{n}\right), \widehat{F}$ being its Fourier transform.

By the Heisenberg inequality on $\mathbb{R}^{n}$, we have

$$
\begin{aligned}
\frac{\|F\|_{2}^{2}}{4 \pi} \leq & \left(\int_{\mathbb{R}^{n}}\left|x_{1}\right|^{2}\left|F\left(x_{1}, \ldots, x_{n}\right)\right|^{2} d x_{1} \cdots d x_{n}\right)^{1 / 2} \\
& \times\left(\int_{\mathbb{R}^{n}}\left|\xi_{1}\right|^{2}\left|\widehat{F}\left(\xi_{1}, \xi_{2}, \ldots, \xi_{n}\right)\right|^{2} d \xi_{1} d \xi_{2} \cdots d \xi_{n}\right)^{1 / 2} .
\end{aligned}
$$

But

$$
\begin{aligned}
\|F\|_{2}^{2} & =\int_{\mathbb{R}^{n}}\left|F\left(x_{1}, \ldots, x_{n}\right)\right|^{2} d x_{1} \cdots d x_{n} \\
& =\int_{\mathbb{R}^{n}}\left|(f \circ \exp )\left(\sum_{i=1}^{n} x_{i} X_{i}\right)\right|^{2} d x_{1} \cdots d x_{n}=\int_{G}|f(x)|^{2} d x=\|f\|_{2}^{2} .
\end{aligned}
$$


Combining (4.2), (4.3), and (4.4), we get

$$
\frac{\|f\|_{2}^{2}}{4 \pi} \leq\left(\int_{G}\|x\|^{2}|f(x)|^{2} d x\right)^{1 / 2}\left(\int_{\mathcal{W}}\|\xi\|^{2}\left\|\pi_{\xi}(f)\right\|_{\mathrm{HS}}^{2} \frac{1}{|h(\xi)|} d \xi\right)^{1 / 2} .
$$

Now, as in the proof of Theorem 3.2, applications of Hölder's inequality give

$$
\int_{G}\|x\|^{2}|f(x)|^{2} d x \leq\left(\int_{G}\|x\|^{2 a}|f(x)|^{2} d x\right)^{\frac{1}{a}}\left(\|f\|_{2}^{2}\right)^{1-\frac{1}{a}}
$$

and

$$
\begin{aligned}
& \int_{\mathcal{W}}\|\xi\|^{2}\left\|\pi_{\xi}(f)\right\|_{\mathrm{HS}}^{2} \frac{1}{|h(\xi)|} d \xi \\
& \quad \leq\left(\int_{\mathcal{W}}\|\xi\|^{2 b}\left\|\pi_{\xi}(f)\right\|_{\mathrm{HS}}^{2} \frac{1}{|h(\xi)|^{b}|\operatorname{Pf}(\xi)|^{b-1}} d \xi\right)^{\frac{1}{b}}\left(\|f\|_{2}^{2}\right)^{1-\frac{1}{b}} .
\end{aligned}
$$

Combining (4.5), (4.6), and (4.7), we obtain

$$
\frac{\|f\|_{2}^{\left(\frac{1}{a}+\frac{1}{b}\right)}}{4 \pi} \leq\left(\int_{G}\|x\|^{2 a}|f(x)|^{2} d x\right)^{\frac{1}{2 a}}\left(\int_{\mathcal{W}}\|\xi\|^{2 b}\left\|\pi_{\xi}(f)\right\|_{\mathrm{HS}}^{2} \frac{1}{|h(\xi)|^{b}|\operatorname{Pf}(\xi)|^{b-1}} d \xi\right)^{\frac{1}{2 b}}
$$

Example 4.2 We now list several classes that are included in the above general class.

1. For thread-like nilpotent Lie groups (for details, see [8]), we have $\operatorname{Pf}(\xi)=\xi_{1}$ and

$$
\mathcal{W}=\left\{\xi=\left(\xi_{1}, 0, \xi_{3}, \ldots, \xi_{n-1}, 0\right): \xi_{j} \in \mathbb{R}, \xi_{1} \neq 0\right\}
$$

Also, $\left\|\pi_{\xi}(f)\right\|_{H S}$ is given by

$$
\left\|\pi_{\xi}(f)\right\|_{\mathrm{HS}}^{2}=\frac{1}{\left|\xi_{1}\right|} \int_{\mathbb{R}^{2}}\left|\mathscr{F}(f \circ \exp )\left(\xi_{1}, t, \xi_{3}+Q_{3}, \ldots, \xi_{n-1}+Q_{n-1}, s\right)\right|^{2} d s d t,
$$

where $Q_{j}\left(\xi_{1}, 0, \xi_{3}, \ldots, \xi_{j-1}, t\right)=\sum_{k=1}^{j-1} \frac{1}{k !} \frac{t}{k}_{1}^{k} \xi_{j-k}$, for $3 \leq j \leq n-1$.

Thus, for $h(\xi)=\frac{1}{|\xi 1|}=\frac{1}{|\operatorname{Pf}(\xi)|}$, one obtains the Heisenberg uncertainty inequality

$$
\frac{\|f\|_{2}^{\left(\frac{1}{a}+\frac{1}{b}\right)}}{4 \pi} \leq\left(\int_{G}\|x\|^{2 a}|f(x)|^{2} d x\right)^{\frac{1}{2 a}}\left(\int_{\mathcal{W}}\|\xi\|^{2 b}\left\|\pi_{\xi}(f)\right\|_{\mathrm{HS}}^{2}\left|\xi_{1}\right| d \xi\right)^{\frac{1}{2 b}} .
$$

2. For 2-NPC nilpotent Lie groups (for details, see [9]), let $\{0\}=\mathfrak{g}_{0} \subset \mathfrak{g}_{1} \subset \cdots \subset \mathfrak{g}_{n}=\mathfrak{g}$ be a Jordan-Hölder sequence in $\mathfrak{g}$ such that $\mathfrak{g}_{m}=\mathfrak{z}(g)$ and $\mathfrak{h}=\mathfrak{g}_{n-2}$. Let us consider the ideal $\left[\mathfrak{g}, \mathfrak{g}_{m+1}\right]$ of $\mathfrak{g}$ which is one or two dimensional in $\mathfrak{g}$. We discuss the two cases separately:

(a) $\operatorname{dim}\left[\mathfrak{g}, \mathfrak{g}_{m+1}\right]=2$.

In this case, for every basis $\left\{X_{1}, X_{2}\right\}$ of $\mathfrak{h}$ in $\mathfrak{g}$ and every $Y_{1} \in \mathfrak{g}_{m+1} \backslash \mathfrak{z}(\mathfrak{g})$, the vectors $Z_{1}=\left[X_{1}, Y_{1}\right]$ and $Z_{2}=\left[X_{2}, Y_{1}\right]$ are linearly independent and lie in the center of $\mathfrak{g}$. Assume that $\mathfrak{g}_{1}=\mathbb{R}-\operatorname{span}\left\{Z_{1}\right\}, \mathfrak{g}_{2}=\mathbb{R}-\operatorname{span}\left\{Z_{1}, Z_{2}\right\}$. Let $Z_{3}, \ldots, Z_{m}$ be some vectors such that $\mathfrak{z}(\mathfrak{g})=$ $\mathbb{R}$-span $\left\{Z_{1}, \ldots, Z_{m}\right\}$ and $\mathcal{B}=\left\{Z_{1}, \ldots, Z_{n}\right\}$ a Jordan-Hölder basis of $\mathfrak{g}$ chosen as follows:

(i) $\mathfrak{z}(\mathfrak{g})=\mathbb{R}-\operatorname{span}\left\{Z_{1}, \ldots, Z_{m}\right\}$;

(ii) $\mathfrak{h}=\mathbb{R}-\operatorname{span}\left\{Z_{1}, \ldots, Z_{n-2}\right\}$;

(iii) $\mathfrak{g}=\mathbb{R}-\operatorname{span}\left\{Z_{1}, \ldots, Z_{n-2}, X_{1}=Z_{n-1}, X_{2}=Z_{n}\right\}$ 
For $m_{1}=m+1$ and $m+2 \leq m_{2} \leq n-2$, we denote $Z_{m_{1}}=Z_{m+1}=Y_{1}, Z_{m_{2}}=Y_{2}$. These vectors can be chosen such that $\xi_{1}=\xi\left(\left[X_{1}, Y_{1}\right]\right) \neq 0, \xi_{2,2}=\xi\left(\left[X_{2}, Y_{2}\right]\right) \neq 0$, for all $\xi \in \mathcal{W}$, where

$$
\mathcal{W}=\left\{\xi=\left(\xi_{1}, \xi_{2}, \ldots, \xi_{m}, 0,0, \xi_{m+3}, \xi_{m+4}, \ldots, \xi_{n-2}, 0,0\right): \xi_{j} \in \mathbb{R},|\operatorname{Pf}(\xi)| \neq 0\right\}
$$

Also, we have $\operatorname{Pf}(\xi)=\xi\left(Z_{1}\right) \xi\left(\left[X_{2}, Y_{2}\right]\right)-\xi\left(\left[X_{1}, Y_{2}\right]\right) \xi\left(Z_{2}\right)$ and $\left\|\pi_{\xi}(f)\right\|_{\mathrm{HS}}$ is given by

$$
\begin{aligned}
\left\|\pi_{\xi}(f)\right\|_{\mathrm{HS}}^{2}= & |h(\xi)| \int_{\mathbb{R}^{4}} \mid \mathscr{F}(f \circ \exp )\left(s_{2}, s_{1}, P_{n-2}\left(\xi,-\frac{t_{1}}{\tilde{\xi}_{1,1}},-\frac{t_{2}}{\tilde{\xi}_{2,2}}\right), \ldots,\right. \\
& \left.P_{m+3}\left(\xi,-\frac{t_{1}}{\tilde{\xi}_{1,1}},-\frac{t_{2}}{\tilde{\xi}_{2,2}}\right), t_{2}, t_{1}, \xi_{m}, \ldots, \xi_{1}\right)\left.\right|^{2} d s_{1} d s_{2} d t_{1} d t_{2},
\end{aligned}
$$

where $h$ is the function defined by

$$
h(\xi)=\frac{\left|\xi_{1} \xi_{2,2}\right|^{2}}{\left|\xi_{1} \xi_{2,2}-\xi_{1,2} \xi_{2}\right|^{2}}
$$

$\xi_{i, j}=\xi\left(\left[X_{i}, Y_{j}\right]\right), \tilde{\xi}_{i, j}=\xi\left(\left[X_{i}(\xi), Y_{j}\right]\right)$, and $P_{j}(\xi, t)$ is a polynomial function with respect to the variables $t=\left(t_{1}, t_{2}\right)$ and $\xi_{m+1}, \ldots, \xi_{j}$ and rational in the variables $\xi_{1}, \ldots, \xi_{m}$. Thus, one obtains the Heisenberg uncertainty inequality

$$
\frac{\|f\|_{2}^{\left(\frac{1}{a}+\frac{1}{b}\right)}}{4 \pi} \leq\left(\int_{G}\|x\|^{2 a}|f(x)|^{2} d x\right)^{\frac{1}{2 a}}\left(\int_{\mathcal{W}}\|\xi\|^{2 b}\left\|\pi_{\xi}(f)\right\|_{\mathrm{HS}}^{2} \frac{1}{|h(\xi)|^{b}|\operatorname{Pf}(\xi)|^{b-1}} d \xi\right)^{\frac{1}{2 b}}
$$

(b) $\operatorname{dim}\left[\mathfrak{g}, \mathfrak{g}_{m+1}\right]=1$.

In this case, we have $\operatorname{Pf}(\xi)=\xi\left(\left[X_{1}, Y_{1}\right]\right) \cdot \xi\left(\left[X_{2}, Y_{2}\right]\right)$ and

$$
\begin{aligned}
\mathcal{W}= & \left\{\xi=\left(\xi_{1}, \xi_{2}, \ldots, \xi_{m}, 0, \xi_{m+2}, \ldots, \xi_{m+d+1}, 0, \xi_{m+d+3}, \ldots, \xi_{n-2}, 0,0\right):\right. \\
& \left.\xi_{j} \in \mathbb{R},|\operatorname{Pf}(\xi)| \neq 0\right\} .
\end{aligned}
$$

Also, $\left\|\pi_{\xi}(f)\right\|_{\mathrm{HS}}$ is given by

$$
\begin{aligned}
\left\|\pi_{\xi}(f)\right\|_{\mathrm{HS}}^{2}= & \frac{1}{|\operatorname{Pf}(\xi)|} \int_{\mathbb{R}^{4}} \mid \mathscr{F}(f \circ \exp )\left(s_{2}, s_{1}, P_{n-2}\left(\xi,-\frac{t_{1}}{\xi_{1}},-\frac{t_{2}+R\left(-\frac{t_{1}}{\xi_{1}}, \xi_{1}, \ldots, \xi_{m+d}\right)}{\xi_{2,2}}\right),\right. \\
& \left.\ldots, t_{2}, \ldots, P_{m+2}\left(\xi,-\frac{t_{1}}{\xi_{1}}\right), t_{1}, \xi_{m}, \ldots, \xi_{1}\right)\left.\right|^{2} d s_{1} d s_{2} d t_{1} d t_{2} .
\end{aligned}
$$

Thus, for $h(\xi)=\frac{1}{|\operatorname{Pf}(\xi)|}$, one obtains the Heisenberg uncertainty inequality,

$$
\frac{\|f\|_{2}^{\left(\frac{1}{a}+\frac{1}{b}\right)}}{4 \pi} \leq\left(\int_{G}\|x\|^{2 a}|f(x)|^{2} d x\right)^{\frac{1}{2 a}}\left(\int_{\mathcal{W}}\|\xi\|^{2 b}\left\|\pi_{\xi}(f)\right\|_{\mathrm{HS}}^{2}|\operatorname{Pf}(\xi)| d \xi\right)^{\frac{1}{2 b}} .
$$

3. For connected, simply connected nilpotent Lie groups $G=\exp \mathfrak{g}$ such that $\mathfrak{g}(\xi) \subset[\mathfrak{g}, \mathfrak{g}]$ for all $\xi \in \mathcal{U}$ (for details, see [10]), we consider $S=\left\{j_{1}<\cdots<j_{d}\right\}$ and $T=\left\{t_{1}<\cdots<t_{r}\right\}$ to be the collection of jump and non-jump indices, respectively, with respect to the basis $\mathcal{B}$. We have $j_{d}=n$ and

$$
\mathcal{W}=\left\{\xi=\left(\xi_{1}, \xi_{2}, \ldots, \xi_{n}\right) \in \mathfrak{g}^{*}: \xi_{j_{i}}=0, \text { for } j_{i} \in S \text { with }|\operatorname{Pf}(\xi)| \neq 0\right\}
$$


Also, $\left\|\pi_{\xi}(f)\right\|_{H S}$ is given by

$$
\left\|\pi_{\xi}(f)\right\|_{\mathrm{HS}}^{2}=\frac{\left|\xi\left(\left[X_{j_{1}}, X_{n}\right]\right)\right|}{|\operatorname{Pf}(\xi)|^{2}} \int_{\mathcal{W}}|\mathscr{F}(f \circ \exp )(\xi, w)|^{2} d w
$$

where $\xi=\left(\xi_{t_{i}}\right)_{t_{i} \in T}$ and $w=\left(w_{j_{i}}\right)_{j_{i} \in S}$. Thus, for $h(\xi)=\frac{\left|\xi\left(\left[X_{j_{1}}, X_{n}\right]\right)\right|}{|\operatorname{Pf}(\xi)|^{2}}$, one obtains the Heisenberg uncertainty inequality

$$
\frac{\|f\|_{2}^{\left(\frac{1}{a}+\frac{1}{b}\right)}}{4 \pi} \leq\left(\int_{G}\|x\|^{2 a}|f(x)|^{2} d x\right)^{\frac{1}{2 a}}\left(\int_{\mathcal{W}}\|\xi\|^{2 b}\left\|\pi_{\xi}(f)\right\|_{\mathrm{HS}}^{2} \frac{|\mathrm{Pf}(\xi)|^{b+1}}{\left|\xi\left(\left[X_{j_{1}}, X_{n}\right]\right)\right|^{b}} d \xi\right)^{\frac{1}{2 b}} .
$$

4. For low-dimensional nilpotent Lie groups of dimension less than or equal to 6 (for details, see [11]) except for $G_{6,8}, G_{6,12}, G_{6,14}, G_{6,15}, G_{6,17}$, an explicit form of $\left\|\pi_{\xi}(f)\right\|_{\text {HS }}$ can be obtained. Thus, an explicit Heisenberg uncertainty inequality can be written down.

5. The classes mentioned above are distinct. For instance, $G_{5,5}$ is thread-like nilpotent Lie group, but it does not belong to the class mentioned in item 3. above. Also, $G_{5,3}$ belongs to the class mentioned in item 3. above, but it is not a thread-like nilpotent Lie group.

Competing interests

The authors declare that they have no competing interests.

Authors' contributions

All authors contributed equally to this paper and they read and approved the final manuscript.

\section{Author details}

'Department of Mathematics, Keshav Mahavidyalaya, University of Delhi, H-4-5 Zone, Pitampura, Delhi, 110034, India.

${ }^{2}$ Department of Mathematics, University of Delhi, Delhi, 110007, India.

\section{Acknowledgements}

The second author was supported by R \& D grant of University of Delhi. The authors would like to thank the referees for many valuable suggestions which helped in improving the exposition.

Received: 10 October 2014 Accepted: 12 May 2015 Published online: 28 May 2015

\section{References}

1. Folland, GB, Sitaram, A: The uncertainty principle: a mathematical survey. J. Fourier Anal. Appl. 3(3), $207-238$ (1997)

2. Thangavelu, S: Some uncertainty inequalities. Proc. Indian Acad. Sci. Math. Sci. 100(2), 137-145 (1990)

3. Sitaram, A, Sundari, M, Thangavelu, S: Uncertainty principles on certain Lie groups. Proc. Indian Acad. Sci. Math. Sci. 105, 135-151 (1995)

4. Xiao, J, He, J: Uncertainty inequalities for the Heisenberg group. Proc. Indian Acad. Sci. Math. Sci. 122(4), 573-581 (2012)

5. Sarkar, RP, Thangavelu, S: On the theorems of Beurling and Hardy for the Euclidean motion group. Tohoku Math. J. 57, 335-351 (2005)

6. Kumahara, K, Okamoto, K: An analogue of the Paley-Wiener theorem for the Euclidean motion group. Osaka J. Math. 10, 77-92 (1973)

7. Corwin, L, Greenleaf, FP: Representations of Nilpotent Lie Groups and Their Applications: Part I. Basic Theory and Examples. Cambridge University Press, Cambridge (1990)

8. Kaniuth, E, Kumar, A: Hardy's theorem for simply connected nilpotent Lie groups. Math. Proc. Camb. Philos. Soc. 131, 487-494 (2001)

9. Baklouti, A, Salah, NB: On theorems of Beurling and Cowling-Price for certain nilpotent Lie groups. Bull. Sci. Math. 132, 529-550 (2008)

10. Smaoui, K: Beurling's theorem for nilpotent Lie groups. Osaka J. Math. 48, 127-147 (2011)

11. Nielson, OA: Unitary Representations and Coadjoint Orbits of Low-Dimensional Nilpotent Lie Groups. Queens Papers in Pure and Appl. Math. Queen's University, Kingston (1983) 